\title{
THE ASYMPTOTIC BEHAVIOUR OF SOLUTIONS OF CERTAIN TYPES OF THE DIFFERENTIAL EQUATIONS PARTIALLY SOLVED RELATIVELY TO THE DERIVATIVES WITH A SINGULARITY IN THE ZERO-POINT
}

\section{DIANA LIMANSKA and GALINA SAMKOVA}

Odessa I. I. Mechnikov National University

Ukraine

e-mail: liman.diana@gmail.com

\begin{abstract}
For the systems of ordinary differential equations which are partially resolved relatively to the derivatives in the case of a pole, the theorems on the existence of at least one analytic in the complex domain solution of the Cauchy problem with an additional condition are established. Moreover, the asymptotic behaviour of these solutions in this domain is studied.
\end{abstract}

\section{Introduction}

The theory of behaviour of the solutions of systems of ordinary differential equations near singular points was advanced by Fuchs, Briot, Buouquet, Lyapunov, Poincaré, Painlevé, and other researchers.

2010 Mathematics Subject Classification: 34M30.

Keywords and phrases: ordinary differential equation, pole, Cauchy's problem, complex domain, singularity, asymptotic behaviour, analytic.

Received July 9, 2018; Revised August 17, 2018

(C) 2018 Scientific Advances Publishers 
At the end of the 19th century, Painlevé, together with Fuchs, proved theorems selecting the classes of equations whose solutions do not have moving singular points or essential singularities. Later, Grudo, Jwano [12], Trjitzinsky, Hurukaru, Wasow, Malmquist, Strod and others studied the systems of ordinary differential equations in the complex domain. The analysis of the existence of solutions of the systems of equations unsolved with respect to the derivatives and the asymptotic behaviour of these solutions is a separate problem. Specific types of systems unsolved with respect to the derivatives were investigated in the complex plane by Jwano [12], Song Guk et al. [13], Gromak, and other researchers.

One of the methods of researching the systems of differential equations which are not resolved relatively to the derivatives in the realvalued domain was suggested by Grabovskaya [1], Grabovskaya and Diblic [2], and Diblic [11]. It was developed in the complex domain in the articles of Samkova [6], Samkova and Sharay [7], Sharay and Samkova [9], Shkil et al. [10], Michalenko, Limanska [4], Limanska and Samkova $[3,5]$ and others. The current article is a continuation of the researching of the systems of differential equations that are not resolved relatively to the derivatives in the complex domain.

Let us consider the system of ordinary differential equations

$$
A(z) Y^{\prime}=B(z) Y+f\left(z, Y, Y^{\prime}\right)
$$

where matrices $A, B: D_{1} \rightarrow \mathbb{C}^{p \times n}, D_{1}=\left\{z \in \mathbb{C}:|z|<R_{1}, R_{1}>0\right\}$, matrices $A(z), B(z)$ are analytic in the domain $D_{10}, D_{10}=D_{1} \backslash\{0\}$, the pencil of matrices $A(z) \lambda-B(z)$ is singular on the condition that $z \rightarrow 0$, vector function $f: D_{1} \times G_{1} \times G_{2} \rightarrow \mathbb{C}^{p}$, where domains $G_{k} \subset \mathbb{C}^{n}$, $0 \in G_{k}, k=1,2$, vector-function $f\left(z, Y, Y^{\prime}\right)$ is analytic in the domain $D_{1} \times G_{10} \times G_{20}, G_{k 0}=G_{k} \backslash\{0\}, k=1,2$.

Some cases of the analytical solutions existence were previously researched, they were proved to exist on the conditions that the system (1) has square matrices $A(z)$ and $B(z)$, and the point $z=0$ is the 
removable singularity or the pole of the matrix $B(z)[3,4]$. Moreover, the case on the conditions that $p<n$ the system (1) and the point $z=0$ is a pole for matrix $B(z)$ was researched [5].

Let us research the system of ordinary differential equations $\left({ }^{*} 1\right)$ on the conditions that $p<n$ and $\operatorname{rang} A(z)=p$ on the condition that $z \in D_{1}$. Let us introduce function $Y=\operatorname{col}\left(Y_{1} Y_{2}\right), Y_{1}=\operatorname{col}\left(Y_{11}(z)\right.$, $\left.\ldots, Y_{1 p}(z)\right), Y_{2}=\operatorname{col}\left(Y_{21}(z), \ldots, Y_{2 n-p}(z)\right), Y_{1}: D_{1} \rightarrow \mathbb{C}^{p}, Y_{2}: D_{1} \rightarrow \mathbb{C}^{n-p}$. Without restricting the generality, let's assume that matrices $A(z), B(z)$ and vector-function $f\left(z, Y, Y^{\prime}\right)$ take the forms

$$
\begin{aligned}
& A(z)=\left(A_{1}(z) \quad A_{2}(z)\right) ; B(z)=\left(B_{1}(z) \quad B_{2}(z)\right) ; \\
& f\left(z, Y, Y^{\prime}\right)=f^{*}\left(z, Y_{1}, Y_{2}, Y_{1}^{\prime}, Y_{2}^{\prime}\right),
\end{aligned}
$$

$A_{1}: D_{1} \rightarrow \mathbb{C}^{p \times p}, A_{2}: D_{1} \rightarrow \mathbb{C}^{p \times(n-p)}, B_{1}: D_{1} \rightarrow \mathbb{C}^{p \times p}, B_{2}: D_{1} \rightarrow \mathbb{C}^{p \times(n-p)}$, $\operatorname{det} A_{1}(z) \neq 0$ on the condition that $z \in D_{1}, f^{*}: D_{1} \times G_{11} \times G_{12} \times G_{21} \times$ $G_{22} \rightarrow \mathbb{C}^{p}, G_{j 1} \times G_{j 2}=G_{j}, G_{j 1} \subset \mathbb{C}^{p}, G_{j 2} \subset \mathbb{C}^{n-p}, j=1,2$.

Let us suppose that matrices $A_{1}^{-1}(z) A_{2}(z), A_{1}^{-1}(z) B_{2}(z)$ are analytic in the domain $D_{10}$ and have removable singularity in the point $z=0$, and matrix $A_{1}^{-1}(z) B_{1}(z)$ is analytic in the domain $D_{10}$ and have a pole of order $r$ in the point $z=0, f^{*}\left(z, Y_{1}, Y_{2}, Y_{1}^{\prime}, Y_{2}^{\prime}\right)$ is analytic vectorfunction in the domain $D_{1} \times G_{11} \times G_{12} \times G_{21} \times G_{22}$.

Let us introduce

$$
A_{1}^{-1}(z) B_{1}(z)=z^{-r} P(z)
$$

$A_{1}^{-1}(z) B_{2}(z) Y_{2}-A_{1}^{-1}(z) A_{2}(z) Y_{2}^{\prime}+A_{1}^{-1}(z) f^{*}\left(z, Y_{1}, Y_{2}, Y_{1}^{\prime}, Y_{2}^{\prime}\right)=F^{*}\left(z, Y_{1}, Y_{2}, Y_{1}^{\prime}, Y_{2}^{\prime}\right)$,

then the system (1) may be written as

$$
Y_{1}^{\prime}=z^{-r} P(z) Y_{1}+F^{*}\left(z, Y_{1}, Y_{2}, Y_{1}^{\prime}, Y_{2}^{\prime}\right),
$$


where $P: D_{1} \rightarrow \mathbb{C}^{p \times p}, P(z)$ is analytic matrix in the domain $D_{1}$, $F^{*}\left(z, Y_{1}, Y_{2}, Y_{1}^{\prime}, Y_{2}^{\prime}\right)$ is analytic vector-function in the domain $D_{1} \times$ $G_{11} \times G_{12} \times G_{21} \times G_{22}$.

By $H_{u}^{n-p}$ we basically mean class of $(n-p)$-dimensional analytic in the domain $D_{10}$ functions that have the pole of $u$-order in the point $=0$, $u \in \mathbb{N}$.

Let us consider the system (3) for arbitrary fixed vector-function $Y_{2} \in H_{u}^{n-p}$. Then the function $Y_{2}=Y_{2}(z)$ may be written as convergent power series on the condition that $z \in D_{1}$ :

$$
Y_{2}(z)=z^{-u} Y_{2}^{*}(z)=\sum_{k=0}^{\infty} C_{k} z^{k-u}
$$

where $Y_{2}^{*}(z)$ is analytic vector-function in the domain $D_{1}$, that $Y_{2}^{*}(0) \neq 0, C_{k} \in \mathbb{C}^{n-p}, k=0,1,2, \ldots, C_{0} \neq 0$.

Because of $F^{*}\left(z, Y_{1}, Y_{2}, Y_{1}^{\prime}, Y_{2}^{\prime}\right)$ is analytic vector-function in the domain $D_{1} \times G_{11} \times G_{12} \times G_{21} \times G_{22}$, then $F^{*}\left(z, Y_{1}, Y_{2}, Y_{1}^{\prime}, Y_{2}^{\prime}\right)$ may be submitted in convergent series about the point $(0,0,0,0,0)$

$$
F^{*}\left(z, Y_{1}, Y_{2}, Y_{1}^{\prime}, Y_{2}^{\prime}\right)=\sum_{\substack{a+|j|+|k|+|b|+|d|=1 \\|k|+|b|+|d| \neq 0}}^{\infty} C_{a j k b d} z^{a} Y_{1}^{j} Y_{2}^{k}\left(Y_{1}^{\prime}\right)^{b}\left(Y_{2}^{\prime}\right)^{d}
$$

where $\quad C_{a j k b d} \in \mathbb{C}^{p}, \beta=\left(\beta_{1}, \ldots, \beta_{p}\right),|\beta|=\beta_{1}+\cdots+\beta_{p},(\Upsilon)^{\beta}=\left(\Upsilon_{1}\right)^{\beta_{1}} \ldots$ $\left(\Upsilon_{p}\right)^{\beta_{p}},\left(j, Y_{1}\right),\left(b, Y_{1}^{\prime}\right) \in\left\{(\beta, \Upsilon): \beta_{j} \in \mathbb{N}, j=\overline{1, p}, \Upsilon: D_{1} \rightarrow \mathbb{C}^{p}\right\}$, $\varsigma=\left(\varsigma_{1}, \ldots, \varsigma_{n-p}\right),|\varsigma|=\varsigma_{1}+\ldots+\varsigma_{n-p},(\psi)^{\varsigma}=\left(\psi_{1}\right)^{\varsigma_{1}} \ldots\left(\psi_{(n-p)}\right)^{\varsigma_{n-p}} \in\left(k, Y_{2}\right)$, $\left(d, Y_{2}^{\prime}\right) \in\left\{(\varsigma, \psi): \varsigma_{j} \in \mathbb{N}, j=\overline{1, n-p}, \psi: D_{1} \rightarrow \mathbb{C}^{n-p}\right\}$. 
Let us assume that there exist such $q \in \mathbb{N}$ and $s \in \mathbb{N}$ that:

(1) for some $a_{0} \in \mathbb{N}, j_{0}=\left(j_{01}, \ldots, j_{0 p}\right), j_{0 h} \in \mathbb{N} \cup\{0\}, h=\overline{1, p}$, $b_{0}=\left(b_{01}, \ldots, b_{0 p}\right), b_{0 h} \in \mathbb{N} \cup\{0\}, h=\overline{1, p}$, on the condition $|k|=q,|d|=s$, $C_{a_{0} j_{0} k b_{0} d} \neq 0$

(2) for and $h, m \in \mathbb{N}$, and $\mu=1,2, \ldots, n-p, c=1,2, \ldots, n-p$, $C_{a_{j}\left(k+h e_{\mu}\right) b\left(d+m e_{c}\right)}=0$,

where $e_{\mu}$ is $(n-p)$-dimensional and $\mu$ orthogonal unit vector.

Therefore, the summands of the power series expansion of function $F^{*}$ in the domain of point $(0,0,0,0,0)$ containing the maximum powers of vector-functions $Y_{2}$ and $Y_{2}^{\prime}$ and with non-zero coefficients, may take the form

$C_{a j k b d} z^{a} Y_{1}^{j} Y_{2}^{k}\left(Y_{1}^{\prime}\right)^{b}\left(Y_{2}^{\prime}\right)^{d}=C_{a j k b d} z^{a-u q-(u+1) s} Y_{1}^{j}\left(Y_{2}^{*}\right)^{k}\left(Y_{1}^{\prime}\right)^{b}\left(z Y_{2}^{*}-r Y_{2}^{*}\right)^{d}$,

on the condition that $a=0,1,2, \ldots,|j|=0,1,2, \ldots,|b|=0,1,2, \ldots$, $|k|=q,|d|=s$. At least there are such summands on the condition that $a=a_{0}, j=j_{0}, b=b_{0}$.

Two logical cases are possible:

(1) $a-u q-(u+1) s \geq 0$. Then for the arbitrary fixed function $Y_{2} \in H_{u}^{n-p}, F^{*}\left(z, Y_{1}, Y_{2}, Y_{1}^{\prime}, Y_{2}^{\prime}\right)=F\left(z, Y_{1}, Y_{1}^{\prime}\right)$, where $F\left(z, Y_{1}, Y_{1}^{\prime}\right)$ is analytic function in the point $(0,0,0)$, and the system (3) is reduced to the system

$$
z^{r} Y_{1}^{\prime}=P(z) Y_{1}+z^{r} F\left(z, Y_{1}, Y_{1}^{\prime}\right)
$$


We research the questions of the analytic solutions existence of the system (5) that satisfy the initial condition

$$
Y_{1}(z) \rightarrow 0 \text { on the condition that } z \rightarrow 0, z \in D_{10},
$$

and additional condition

$$
Y_{1}^{\prime}(z) \rightarrow 0 \text { on the condition that } z \rightarrow 0, z \in D_{10} .
$$

Let us adapt the results of the Theorem 1 from the article ([3], стр. 22 ), we find the sufficient conditions of existence analytic solutions of Cauchy's problem (5)-(6) with the additional condition (7).

(2) $a-u q-(u+1) s<0$. Let us introduce $l=-a+u q+(u+1) s$, then the vector-function $F^{*}\left(z, Y_{1}, Y_{2}, Y_{1}^{\prime}, Y_{2}^{\prime}\right)$ may take the form

$$
\begin{aligned}
F^{*}\left(z, Y_{1}, Y_{2}, Y_{1}^{\prime}, Y_{2}^{\prime}\right) & =z^{-l} \sum_{\alpha+|j|+|k|+|b|+|d|=0}^{\infty} C_{a j k b d} z^{a} Y_{1}^{j}\left(Y_{2}^{*}\right)^{k}\left(Y_{1}^{\prime}\right)^{b}\left(z Y_{2}^{*}-r Y_{2}^{*}\right)^{d} \\
& =z^{-l} \cdot \check{F}\left(z, Y_{1}, Y_{2}^{*}, Y_{1}^{\prime}, Y_{2}^{*}\right)
\end{aligned}
$$

where $\check{F}\left(z, Y_{1}, Y_{2}^{*}, Y_{1}^{\prime}, Y_{2}^{*^{\prime}}\right)$ is analytic vector-function in the domain $D_{1} \times G_{11} \times G_{12} \times G_{21} \times G_{22}$. On the condition that $Y_{2} \in H_{u}^{n-p}$, let's assume that $\check{F}\left(z, Y_{1}, Y_{2}^{*}, Y_{1}^{\prime}, Y_{2}^{*}\right)=F\left(z, Y_{1}, Y_{1}^{\prime}\right)$, moreover $F(0,0,0)=0$, and the system (3) on the condition that $r_{0}=\max (l, r)$ is reduced to the system

$$
z^{r_{0}} Y_{1}^{\prime}=z^{r_{0}-r} P(z) Y_{1}+z^{r_{0}-l} F\left(z, Y_{1}, Y_{1}^{\prime}\right)
$$




\section{Introduction of Some Intermediary Notation}

For arbitrary fixed $t_{1}>0, \nu_{1}, \nu_{2} \in \mathbb{R}, \nu_{1}<\nu_{2}$, we introduce auxiliary sets: $\check{I}=\left\{(t, \nu) \in \mathbb{R}^{2}: t \in\left(0, t_{1}\right), \nu \in\left(\nu_{1}, \nu_{2}\right)\right\} ; L_{\nu_{0}}\left(t_{1}\right)=\{(t, \nu)$ $\left.\in \mathbb{R}^{2}: t \in\left(0, t_{1}\right), v=\nu_{0} \in\left(\nu_{1}, \nu_{2}\right)\right\}, \nu_{0}$ is a fixed number. For arbitrary fixed $t_{0} \in\left(0, t_{1}\right), 0_{t_{1}}\left(t_{0}\right)=\left\{(t, v) \in \mathbb{R}^{2}: t=t_{0}, \nu \in\left(\nu_{1}, \nu_{2}\right)\right\}$.

On the condition that $z=z(t, v)=t e^{i \nu}$, we associate the set $\check{I} \subset \mathbb{R}^{2}$ with the set $I \subset \mathbb{C}: I=\left\{z=t e^{i \nu} \in \mathbb{C}: t \in\left(0, t_{1}\right), \nu \in\left(\nu_{1}, \nu_{2}\right)\right\}$.

Definition 1. Let functions $p, g: \check{I} \rightarrow[0,+\infty)$. We say that a function $p(t, v)$ possesses the property $Q_{1}$ with respect to the function $g(t, v)$ on the condition that $\nu=\nu_{0} \in\left(\nu_{1}, \nu_{2}\right)$, if $p\left(t, \nu_{0}\right)$ is the function of higher order of smallness with respect to the function $g\left(t, v_{0}\right)$ on the condition that $t \rightarrow+0$.

Definition 2. Let functions $p, g: \check{I} \rightarrow[0,+\infty)$. We say that a function $p(t, v)$ possesses the property $Q_{2}$ with respect to the function $g(t, v)$, if there exist $C_{1} \geq 0, C_{2} \geq 0$, such that the inequality

$$
C_{1} \cdot g(t, \nu) \leq p(t, \nu) \leq C_{2} \cdot g(t, \nu)
$$

is true on the set $\check{I}$.

We introduce auxiliary vector functions as follows:

$$
\begin{aligned}
& \varphi^{(0)}(z)=\left(\varphi_{1}^{(0)}(z), \ldots, \varphi_{p}^{(0)}(z)\right), \varphi^{(0)}: I \rightarrow \mathbb{C}^{p}, \psi^{(0)}(t, \nu)=\left(\psi_{1}^{(0)}(t, \nu), \ldots,\right. \\
& \left.\psi_{p}^{(0)}(t, \nu)\right), \psi_{j}^{(0)}: \check{I} \rightarrow[0 ;+\infty), j=\overline{1, p} \text {, on the condition that } z=z(t, \nu) \\
& =t e^{i \nu}, \psi_{j}^{(0)}(t, \nu)=\left|\varphi_{j}^{(0)}(z(t, \nu))\right|, j=\overline{1, p} .
\end{aligned}
$$


Let us choose the vector function $\varphi^{(0)}(z)$ that is analytic on the set $I$ and, for any $z \in I$, the following conditions are true for the vector-functions $\quad \psi_{j}^{(0)}(t, v): \psi_{j}^{(0)}(t, v)>0 ;\left(\psi_{j}^{(0)}(t, v)\right)_{t}^{\prime}>0 ;\left(\psi_{j}^{(0)}(t, v)\right)_{\nu}^{\prime} \geq 0$; $\psi_{j}^{(0)}(+0, v)=0,\left(\psi_{j}^{(0)}(+0, v)\right)_{t}^{\prime}=0, j=\overline{1, p}$ uniformly in $v \in\left(v_{1}, \nu_{2}\right)$.

\section{System (9) on the Set $L_{\nu_{0}}\left(t_{1}\right)$}

Consider the system (9) on the segment $L_{\nu_{0}}\left(t_{1}\right)$ for any fixed $\nu_{0} \in\left(\nu_{1}, \nu_{2}\right)$.

For $z=z\left(t, \nu_{0}\right)=t e^{i_{v_{0}}}$ we represent each function and matrix in system (9) in the algebraic form by separating the real and imaginary parts and introducing the following notation: $Y_{1}\left(z\left(t, \nu_{0}\right)\right)=\tilde{Y}_{1}(t), \tilde{Y}_{1}(t)=$ $\tilde{Y}_{11}(t)+i \tilde{Y}_{12}(t), \tilde{Y}_{1 j}(t)=\operatorname{col}\left(\tilde{Y}_{1 j 1}(t), \ldots, \widetilde{Y}_{1 j p}(t)\right), j=1,2, P\left(z\left(t, \nu_{0}\right)\right)=\left\|\tilde{p}_{j k}(t)\right\|_{j, k=1}^{p}$ $=\widetilde{P}_{1}(t)+i \widetilde{P}_{2}(t), \widetilde{P}_{s}(t)=\left\|\tilde{p}_{j k s}(t)\right\|_{j, k=1}^{p}, s=1,2, \quad$ where $\quad \tilde{p}_{j k}(t)=\tilde{p}_{j k 1}(t)+$ $i \widetilde{p}_{j k 2}(t), j, k=\overline{1, p}, F\left(z\left(t, v_{0}\right), Y_{1}\left(z\left(t, v_{0}\right)\right), Y_{1}^{\prime}\left(z\left(t, v_{0}\right)\right)\right)=\widetilde{F}\left(t, \tilde{Y}_{1}, \tilde{Y}_{1}^{\prime}\right)$, $\widetilde{F}\left(t, \widetilde{Y}_{1}, \widetilde{Y}_{1}^{\prime}\right)=\operatorname{col}\left(\widetilde{F}_{1}\left(t, \widetilde{Y}_{1}, \widetilde{Y}_{1}^{\prime}\right), \ldots, \widetilde{F}_{p}\left(t, \widetilde{Y}_{1}, \tilde{Y}_{1}^{\prime}\right)\right), \widetilde{F}_{j}\left(t, \widetilde{Y}_{1}, \widetilde{Y}_{1}^{\prime}\right)=\widetilde{F}_{1 j}\left(t, \widetilde{Y}_{1}, \tilde{Y}_{1}^{\prime}\right)+$ $i \widetilde{F}_{2 j}\left(t, \widetilde{Y}_{1}, \widetilde{Y}_{1}^{\prime}\right), j=\overline{1, p}$.

System (9) can be reduced to the form

$$
\begin{aligned}
& t^{r_{0}}\left(\widetilde{Y}_{11}^{\prime}+i \tilde{Y}_{12}^{\prime}\right)=t^{r_{0}-r}\left(\widetilde{P}_{1}+i \widetilde{P}_{2}\right)\left(\tilde{Y}_{11}+i \widetilde{Y}_{12}\right) e^{(1-r) i \nu_{0}} \\
& \quad+t^{r_{0}-l} e^{(1-l) i \nu_{0}}\left(\operatorname{Re} \widetilde{F}\left(t, \widetilde{Y}_{1}, \widetilde{Y}_{1}^{\prime}\right)+i \operatorname{lm} \widetilde{F}\left(t, \widetilde{Y}_{1}, \widetilde{Y}_{1}^{\prime}\right)\right) .
\end{aligned}
$$


We now introduce the following matrices and a vector-function

$$
\begin{gathered}
\widetilde{P}(t)=\left(\begin{array}{cc}
\widetilde{P}_{1}(t) & -\widetilde{P}_{2}(t) \\
\widetilde{P}_{2}(t) & \widetilde{P}_{1}(t)
\end{array}\right), \widetilde{Q}_{1}\left(\nu_{0}\right)=\left(\begin{array}{cc}
\cos \left((r-1) \nu_{0}\right) E & \sin \left((r-1) \nu_{0}\right) E \\
-\sin \left((r-1) \nu_{0}\right) E & \cos \left((r-1) \nu_{0}\right) E
\end{array}\right), \\
\widetilde{Q}_{2}\left(\nu_{0}\right)=\left(\begin{array}{cc}
\cos \left((l-1) \nu_{0}\right) E & \sin \left((l-1) \nu_{0}\right) E \\
-\sin \left((l-1) \nu_{0}\right) E & \cos \left((l-1) \nu_{0}\right) E
\end{array}\right) \\
\widetilde{f}\left(t, \widetilde{Y}_{11}, \widetilde{Y}_{12}, \widetilde{Y}_{11}^{\prime}, \widetilde{Y}_{12}^{\prime}\right)=\operatorname{col}\left(\widetilde{F}_{11} \ldots \widetilde{F}_{1 p} \widetilde{F}_{21} \ldots \widetilde{F}_{2 p}\right)
\end{gathered}
$$

where $E$ is the identity $p \times p$ matrix.

We now equate the real and imaginary parts of the indicated vector functions on the left- and right-hand sides of system (10). This enables us to rewrite system (10) in the form

$$
t^{r_{0}}\left(\begin{array}{c}
\tilde{Y}_{11}^{\prime}(t) \\
\tilde{Y}_{12}^{\prime}(t)
\end{array}\right)=t^{r_{0}-r} \widetilde{P}(t) \widetilde{Q}_{1}\left(v_{0}\right)\left(\begin{array}{c}
\tilde{Y}_{11}(t) \\
\tilde{Y}_{12}(t)
\end{array}\right)+t^{r_{0}-l} \widetilde{Q}_{2}\left(v_{0}\right) \tilde{f}\left(t, \tilde{Y}_{11}, \tilde{Y}_{12}, \tilde{Y}_{11}^{\prime}, \tilde{Y}_{12}^{\prime}\right)
$$

Hence, we have reduced system (9) along the segment $L_{\nu_{0}}\left(t_{1}\right)$ for any fixed $\nu_{0} \in\left(\nu_{1}, \nu_{2}\right)$ to system of differential equations (11).

\section{System (9) on the Set $O_{t_{1}}\left(t_{0}\right)$}

Consider system (9) along arc $O_{t_{1}}\left(t_{0}\right)$ of the circle for any fixed $t_{0} \in\left(0, t_{1}\right)$.

For $z=z\left(t_{0}, \nu\right)=t_{0} e^{i \nu}$, we represent each function and matrix in system (9) in the algebraic form by separating the real and imaginary parts and introducing the following notation: 


$$
\begin{aligned}
& Y_{1}\left(z\left(t_{0}, \nu\right)\right)=\widehat{Y}_{1}(\nu), \widehat{Y}_{1}(\nu)=\widehat{Y}_{11}(\nu)+i \widehat{Y}_{12}(\nu), \hat{Y}_{1 j}(\nu)=\operatorname{col}\left(\widehat{Y}_{1 j 1}(\nu), \ldots, \widehat{Y}_{1 j p}(\nu)\right), \\
& j=1,2, P\left(z\left(t_{0}, \nu\right)\right)=\left\|\hat{p}_{j k}(\nu)\right\|_{j, k=1}^{p}=\widehat{P}_{1}(v)+i \widehat{P}_{2}(v), \widehat{P}(v)=\left\|\hat{p}_{j k s}(\nu)\right\|_{j, k=1}^{p}, \\
& s=1,2, \quad \text { where } \quad \hat{p}_{j k}(v)=\hat{p}_{j k 1}(v)+i \hat{p}_{j k 2}(\nu), j, k=\overline{1, p}, F\left(z\left(t_{0}, \nu\right), Y_{1}(z\right. \\
& \left.\left.\left(t_{0}, \nu\right)\right), Y_{1}^{\prime}\left(z\left(t_{0}, v\right)\right)\right)=\widehat{F}\left(\nu, \hat{Y}_{1}, \widehat{Y}_{1}^{\prime}\right), \widehat{F}\left(\nu, \hat{Y}_{1}, \hat{Y}_{1}^{\prime}\right)=\operatorname{col}\left(\widehat{F}_{1}\left(\nu, \hat{Y}_{1}, \hat{Y}_{1}^{\prime}\right), \ldots,\right. \\
& \left.\widehat{F}_{p}\left(\nu, \widehat{Y}_{1}, \hat{Y}_{1}^{\prime}\right)\right), \widehat{F}_{j}\left(\nu, \hat{Y}_{1}, \hat{Y}_{1}^{\prime}\right)=\widehat{F}_{1 j}\left(\nu, \hat{Y}_{1}, \hat{Y}_{1}^{\prime}\right)+i \widehat{F}_{2 j}\left(\nu, \widehat{Y}_{1}, \hat{Y}_{1}^{\prime}\right), j=\overline{1, p}
\end{aligned}
$$

The system (9) can be reduced, for $=z\left(t_{0}, v\right)=t_{0} e^{i v}$, to the form

$$
\begin{aligned}
& t_{0}^{r_{0}-1}\left(\widehat{Y}_{11}^{\prime}+i \widehat{Y}_{12}^{\prime}\right)=i t_{0}^{r_{0}-r}\left(\widehat{P}_{1}+i \widehat{P}_{2}\right)\left(\widehat{Y}_{11}+i \widehat{Y}_{12}\right) e^{(1-r) i \nu}+ \\
& \quad+i t_{0}^{r_{0}-l} e^{(1-l) i v}\left(\operatorname{Re} \widehat{F}\left(\nu, \widehat{Y}_{1}, \widehat{Y}_{1}^{\prime}\right)+i \operatorname{lm} \widehat{F}\left(\nu, \widehat{Y}_{1}, \widehat{Y}_{1}^{\prime}\right)\right)
\end{aligned}
$$

We now introduce the following matrices and a vector function:

$$
\begin{gathered}
\widehat{P}(\nu)=\left(\begin{array}{cc}
\widehat{P}_{1}(\nu) & -\widehat{P}_{2}(\nu) \\
\widehat{P}_{2}(\nu) & \widehat{P}_{1}(\nu)
\end{array}\right), \widehat{Q}_{1}(\nu)=\left(\begin{array}{cc}
\sin ((r-1) \nu) E & -\cos ((r-1) \nu) E \\
\cos ((r-1) \nu) E & \sin ((r-1) \nu) E
\end{array}\right), \\
\widehat{Q}_{2}(\nu)=\left(\begin{array}{cc}
\sin ((l-1) \nu) E & -\cos ((l-1) \nu) E \\
\cos ((l-1) \nu) E & \sin ((l-1) \nu) E
\end{array}\right) \\
\hat{f}\left(\nu, \widehat{Y}_{11}, \widehat{Y}_{12}, \widehat{Y}_{11}^{\prime}, \widehat{Y}_{12}^{\prime}\right)=\operatorname{col}\left(\widehat{F}_{11} \ldots \widehat{F}_{1 p} \widehat{F}_{21} \ldots \widehat{F}_{2 p}\right) .
\end{gathered}
$$

Equating the real and imaginary parts of the indicated vector functions on the left- and right-hand sides of system (12), we reduce system (12) to the form

$$
t_{0}^{r_{0}-1}\left(\begin{array}{l}
\widehat{Y}_{11}^{\prime}(v) \\
\widehat{Y}_{12}^{\prime}(v)
\end{array}\right)=t_{0}^{r_{0}-r} \widehat{P}(\nu) \widehat{Q}_{1}(\nu)\left(\begin{array}{l}
\widehat{Y}_{11}(v) \\
\widehat{Y}_{12}(v)
\end{array}\right)+t_{0}^{r_{0}-l} \widehat{Q}_{2}(v) \hat{f}\left(v, \widehat{Y}_{11}, \widehat{Y}_{12}, \widehat{Y}_{11}^{\prime}, \widehat{Y}_{12}^{\prime}\right)
$$


Thus, we have reduced system (9) along arc $O_{t_{1}}\left(t_{0}\right)$ of the circle to the system of real differential equations (13) for any fixed $t_{0} \in\left(0, t_{1}\right)$.

\section{Some Classes of Functions and the Properties of System}

Definition 4. We say that the matrix $P(z)$ has the property $S_{3}$ with respect to the vector-function $\varphi^{(0)}(z)$, if the following conditions are satisfied:

(1) Functions $t^{r_{0}}\left(\psi_{j}^{(0)}(z(t, v))\right)^{\prime} t$ have the property $Q_{1}$ with respect to functions $t^{r_{0}-r}\left|\tilde{p}_{j j}(t)\right| \psi_{j}^{(0)}(z(t, v)), j=\overline{1, p}$, on the conditions that $v=v_{0}$ $\in\left(\nu_{1}, \nu_{2}\right)$.

(2) Functions $t^{r_{0}-1}\left(\psi_{j}^{(0)}(t, v)\right)_{\nu}^{\prime}$ have the property $Q_{2}$ with respect to functions $t^{r_{0}-r}\left|\hat{p}_{j j}(\nu)\right| \psi_{j}^{(0)}(t, v), j=\overline{1, p}$.

(3) Functions $t^{r_{0}-r}\left|\widetilde{p}_{j k}(t)\right| \psi_{k}^{(0)}(t, v)$ have the property $Q_{1}$ with respect to functions $t^{r_{0}}\left(\psi_{j}^{(0)}(t, \nu)\right)_{\nu}^{\prime}, j, k=\overline{1, p}, j \neq k$, on the conditions that $\nu=\nu_{0} \in\left(\nu_{1}, \nu_{2}\right)$.

(4) Functions $t^{r_{0}-r}\left|\hat{p}_{j k}(\nu)\right| \psi_{k}^{(0)}(t, v)$ on the conditions that $Q_{2}$ with respect to functions $t^{r_{0}-1}\left(\psi_{j}^{(0)}(t, \nu)\right)_{\nu}^{\prime}, j, k=\overline{1, p}, j \neq k$.

Denote the sets

$$
\begin{array}{r}
\widetilde{\Omega}\left(\delta, \varphi^{(0)}\left(z\left(t, \nu_{0}\right)\right)\right)=\left\{\left(t, \tilde{Y}_{11}, \tilde{Y}_{12}\right): t \in\left(0, t_{1}\right), \tilde{Y}_{11 j}^{2}+\tilde{Y}_{12 j}^{2}<\delta_{j}^{2}\left(\psi_{j}^{(0)}\left(t, \nu_{0}\right)\right)^{2}\right. \\
j=\overline{1, p}\}, \nu_{0} \text { is fixed on }\left(\nu_{1}, \nu_{2}\right) ;
\end{array}
$$




$$
\begin{array}{r}
\widehat{\Omega}\left(\tau, \varphi^{(0)}\left(z\left(t_{0}, \nu\right)\right)\right)=\left\{\left(\nu, \hat{Y}_{11}, \hat{Y}_{12}\right): v \in\left(\nu_{1}, \nu_{2}\right), \widehat{Y}_{11 j}^{2}+\widehat{Y}_{12 j}^{2}<\tau_{j}^{2}\left(\psi_{j}^{(0)}\left(t_{0}, \nu\right)\right)^{2},\right. \\
j=\overline{1, p}\}, t_{0} \text { is fixed on }\left(0, t_{1}\right),
\end{array}
$$

where $\delta=\left(\delta_{1}, \ldots, \delta_{p}\right), \tau=\left(\tau_{1}, \ldots, \tau_{p}\right), \delta_{j}, \tau_{j} \in \mathbb{R} \backslash\{\mathbf{0}\}, j=\overline{1, p}$.

Definition 5. We say that the vector-function $F\left(z, Y_{1}, Y_{1}^{\prime}\right)$ possesses the property $M_{3}$ with respect to the vector-function $\varphi^{(0)}(z)$, if the following conditions are satisfied:

(1) For $\left(t, \tilde{Y}_{11}, \tilde{Y}_{12}\right) \in \widetilde{\Omega}\left(\delta, \varphi^{(0)}\left(z\left(t, v_{0}\right)\right)\right)$ functions $t^{r_{0}-l} \widetilde{F}_{k j}\left(t, \widetilde{Y}_{11}, \widetilde{Y}_{12}\right.$, $\left.\tilde{Y}_{11}^{\prime}, \tilde{Y}_{12}^{\prime}\right)$ have the property $Q_{1}$ with respect to functions $t^{r_{0}-r}\left|\widetilde{p}_{j j}(z(t, \nu))\right| \cdot \psi_{j}^{(0)}(t, \nu), j=\overline{1, p}, k=1,2 \quad$ on the conditions that $\nu=\nu_{0} \in\left(\nu_{1}, \nu_{2}\right)$.

(2) $\quad$ For $\quad\left(\nu, \widehat{Y}_{11}, \widehat{Y}_{12}\right) \in \widehat{\Omega}\left(\tau, \varphi^{(0)}\left(z\left(t_{0}, \nu\right)\right)\right) \quad$ functions $\quad t^{r_{0}-l} \widehat{F}_{k j}$ $\left(\nu, \hat{Y}_{11}, \hat{Y}_{12}, \hat{Y}_{11}^{\prime}, \hat{Y}_{12}^{\prime}\right)$ have the property $Q_{2}$ with respect to functions $t^{r_{0}-r}\left|\hat{p}_{j j}(z(t, \nu))\right| \cdot \psi_{j}^{(0)}(t, \nu), j=\overline{1, p}, k=1,2$.

Without restricting the generality, let us suppose that $t_{1} \leq R_{1}$. Further, we introduce the following domains $\Lambda_{+. k}\left(t_{1}\right), k \in\{+,-\}$

$$
\begin{gathered}
\Lambda_{+.+}\left(t_{1}\right)=\left\{(t, v): \cos \left((r-1) v+\widetilde{\alpha}_{j j}(t)\right)>0,\right. \\
\left.\sin \left((r-1) \nu+\hat{\alpha}_{j j}(v)\right)>0, j=\overline{1, p}, t \in\left(0, t_{1}\right), v \in\left(v_{1}, \nu_{2}\right)\right\} ; \\
\Lambda_{+.-}\left(t_{1}\right)=\left\{(t, v): \cos \left((r-1) \nu+\tilde{\alpha}_{j j}(t)\right)>0,\right. \\
\left.\sin \left((r-1) \nu+\hat{\alpha}_{j j}(v)\right)<0, j=\overline{1, p}, t \in\left(0, t_{1}\right), v \in\left(v_{1}, \nu_{2}\right)\right\} .
\end{gathered}
$$


Definition 6. We say that system (9) belongs to the class $C_{+. k}$, $k \in\{+,-\}$, if the matrix $P(z)=P\left(t e^{i \nu}\right)$ is such that $(t, \nu) \in \Lambda_{+. k}\left(t_{1}\right)$, $k \in\{+,-\}$.

\section{Main Results}

Let us introduce the following domains $G_{+. k}\left(t_{1}\right)=\{z=z(t, v): 0<$ $\left.|z|<t_{1},(t, \nu) \in \Lambda_{+. k}\left(t_{1}\right)\right\}, k \in\{+,-\}$.

Theorem 1. Suppose that the following conditions are satisfied for system (9):

(1) The matrix $P(z)$ is analytic in the domain $D_{1}$ and has the property $S_{3}$ with respect to the analytic vectorfunction $\varphi^{(0)}(z)$.

(2) The vector-function $F\left(z, Y_{1}, Y_{1}^{\prime}\right)$ is analytic in the domain $D_{1} \times G_{11} \times G_{21}$ and has the property $M_{3}$ with respect to the analytic vector function $\varphi^{(0)}(z)$.

(3) The system (9) belongs to one of the classes $C_{+. k}, k \in\{+,-\}$.

Then, for each $k \in\{+,-\}$ and for some $t^{*} \in\left(0, t_{1}\right)$ solutions of the system (9) $Y_{1}(z)$ exist, satisfying the initial conditions $Y_{1}\left(z_{0}\right)=Y_{10}$ for $z_{0} \in G_{+. k}\left(t^{*}\right), Y_{10} \in\left\{Y_{1}:\left|Y_{1 j}\left(z_{0}\right)\right|<\delta_{j}\left|\varphi_{j}^{(0)}\left(z_{0}\right)\right|, \delta_{j}>0, j=\overline{1, p}\right\}, \quad$ are analytic in the domain $D_{1} \cap G_{+. k}\left(t^{*}\right)$ and these solutions admit the estimate

$$
\left|Y_{1 j}(z)\right|^{2}<\delta_{j}^{2}\left|\varphi_{j}^{(0)}(z)\right|^{2}, j=\overline{1, p} .
$$

Proof. (1) We consider system (11), that is corresponding to the system (9) on the segment $L_{\nu_{0}}\left(t_{1}\right)$ for a fixed value $\nu_{0} \in\left(v_{1}, \nu_{2}\right)$. 
Let us introduce sets

$\widetilde{\Omega_{j}}\left(\delta, \varphi^{(0)}\left(z\left(t, \nu_{0}\right)\right)\right)=\left\{\left(t, \widetilde{Y}_{11}, \widetilde{Y}_{12}\right): \widetilde{Y}_{11 j}^{2}+\widetilde{Y}_{12 j}^{2}<\delta_{j}^{2}\left(\phi_{j}^{(0)}\left(t, \nu_{0}\right)\right)^{2}, t \in\left(0, t_{1}\right)\right\}$, $j=\overline{1, p}$. The set $\widetilde{\Omega}\left(\delta, \varphi^{(0)}\left(z\left(t, v_{0}\right)\right)\right)$ is now regarded as the intersection of the sets $\widetilde{\Omega_{j}}, \widetilde{\Omega}\left(\delta, \varphi^{(0)}\left(z\left(t, v_{0}\right)\right)\right)=\bigcap_{j=1}^{p} \widetilde{\Omega_{j}}\left(\delta, \varphi^{(0)}\left(z\left(t, v_{0}\right)\right)\right)$.

We denote a part of the boundary of the set $\widetilde{\Omega_{j}}, j \in\{1,2, \ldots, p\}$, by $\partial \widetilde{\Omega_{j}}\left(\delta, \varphi^{(0)}\left(z\left(t, v_{0}\right)\right)\right)=\left\{\left(t, \widetilde{Y}_{11}, \widetilde{Y}_{12}\right): \widetilde{Y}_{11 j}^{2}+\widetilde{Y}_{12 j}^{2}=\delta_{j}^{2}\left(\psi_{j}^{(0)}\left(t, v_{0}\right)\right)^{2}, \widetilde{Y}_{11 k}^{2}+\widetilde{Y}_{12 k}^{2}\right.$ $\left.<\delta_{k}^{2}\left(\psi_{k}^{(0)}\left(t, v_{0}\right)\right)^{2}, k=\overline{1, p}, k \neq j, t \in\left(0, t_{1}\right)\right\}$.

We also denote $\widetilde{\Phi_{j}}(t, \widetilde{Y}(t))=\widetilde{Y}_{11 j}^{2}(t)+\widetilde{Y}_{12 j}^{2}(t)-\delta_{j}^{2}\left(\psi_{j}^{(0)}\left(t, \nu_{0}\right)\right)^{2}$, $j \in\{1,2, \ldots, p\}$. Then $\frac{\bar{N}_{j}}{2}$ is the vector of outer normal to the surface $\partial \widetilde{\Omega_{j}}\left(\delta, \varphi\left(z\left(t, v_{0}\right)\right)\right)$ for fixed $j \in\{1, \ldots, p\}$.

Let $\bar{T}$ is a vector field of directions of system (11) at any fixed point $\left(t, \tilde{Y}_{11}(t), \widetilde{Y}_{12}(t)\right) \in \partial \widetilde{\Omega}_{j}\left(\delta, \varphi\left(z\left(t, \nu_{0}\right)\right)\right), j \in\{1, \ldots, p\}$.

According to the condition, the matrix $P(z)$ has the property $S_{3}$ and the vector function $F\left(z, Y_{1}, Y_{1}^{\prime}\right)$ has the property $M_{3}$ with respect to the vector function $\varphi^{(0)}(z)$. Therefore,

$$
\left(t^{r_{0}} \bar{T}, \frac{\bar{N}_{j}}{2}\right) \sim \sqrt{\left(\widetilde{p}_{j j 1}(t)\right)^{2}+\left(\widetilde{p}_{j j 2}(t)\right)^{2}}\left(\cos \left((r-1) \nu_{0}+\widetilde{\alpha}_{j j}(t)\right)\right), j=\overline{1, p},
$$

on the condition that $t \rightarrow+0$.

Since system (9) belongs to one of the classes $C_{+. k}(t, v), k \in\{+,-\}$, there exists $t^{*}$, such that the inequality $t \in\left(0, t^{*}\right)$ holds for $\left(t^{r_{0}} \bar{T}, \frac{\bar{N}_{j}}{2}\right)>0$, 
$j=\overline{1, p}$. Hence, for $t \in\left(0, t^{*}\right)$ the surface $\partial \widetilde{\Omega}\left(\delta, \varphi^{(0)}\left(z\left(t, \nu_{0}\right)\right)\right)$ is a surface without contact for system (11). Moreover, the integral curve enters the domain $\widetilde{\Omega}\left(\delta, \varphi^{(0)}\left(z\left(t, \nu_{0}\right)\right)\right)$ as the variable $t$ decreases.

According to Vazhevsky's topological principle, at least one smooth integral curve of system (11) passes through every point of the set $\widetilde{\Omega}\left(\delta, \varphi^{(0)}\left(z\left(t, \nu_{0}\right)\right)\right) \cup \partial \widetilde{\Omega}\left(\delta, \varphi^{(0)}\left(z\left(t, \nu_{0}\right)\right)\right) \cap\left(t=t^{*}\right)$, and all integral curves of this system passing through the points $\widetilde{\Omega}\left(\delta, \varphi^{(0)}\left(z\left(t, v_{0}\right)\right)\right) \cup \partial \widetilde{\Omega}$ $\left(\delta, \varphi^{(0)}\left(z\left(t, v_{0}\right)\right)\right) \cap\left(t=t^{*}\right)$, stay in the domain $\widetilde{\Omega}\left(\delta, \varphi^{(0)}\left(z\left(t, v_{0}\right)\right)\right)$ for $\left(t, \nu_{0}\right) \in \Lambda_{+, k}\left(t^{*}\right), k \in\{+,-\}, \nu_{0} \in\left(\nu_{1}, \nu_{2}\right)$. Moreover, the following inequalities are true

$$
\left|Y_{1 s j}\left(z\left(t, v_{0}\right)\right)\right|^{2}<\delta_{j}{ }^{2}\left(\psi_{j}^{(0)}\left(t, v_{0}\right)\right)^{2}, j=\overline{1, p}, s=1,2,
$$

on the condition that $\left(t, \nu_{0}\right) \in \Lambda_{+. k}\left(t^{*}\right), k \in\{+,-\}$.

(2) We consider system (13), that is corresponding to the system (9) along arc $O_{t_{1}}\left(t_{0}\right)$ of the circle for fixed $t_{0} \in\left(0, t_{1}\right)$.

Let us introduce sets $\quad \widehat{\Omega}_{j}\left(\tau, \varphi^{(0)}\left(z\left(t_{0}, \nu\right)\right)\right)=$ $\left\{\left(\nu, \hat{Y}_{11}, \widehat{Y}_{12}\right): \widehat{Y}_{11 j}^{2}+\widehat{Y}_{12 j}^{2}<\tau_{j}^{2}\left(\psi_{j}^{(0)}\left(t_{0}, \nu\right)\right)^{2}, \nu \in\left(v_{1}, \nu_{2}\right)\right\}, j=\overline{1, p}$. The set $\widehat{\Omega}\left(\tau, \varphi^{(0)}\left(z\left(t_{0}, \nu\right)\right)\right)$ is now regarded as the intersection of the sets $\widehat{\Omega}\left(\tau, \varphi^{(0)}\left(z\left(t_{0}, \nu\right)\right)\right)=\bigcap_{j=1}^{p} \widehat{\Omega}_{j}\left(\tau, \varphi^{(0)}\left(z\left(t_{0}, \nu\right)\right)\right)$.

We denote a part of the boundary of the set $\widehat{\Omega}_{j}, j \in\{1,2, \ldots, p\}$, by

$$
\begin{aligned}
& \partial \widehat{\Omega}_{j}\left(\tau, \varphi^{(0)}\left(z\left(t_{0}, v\right)\right)\right)=\left\{\left(\nu, \hat{Y}_{11}, \hat{Y}_{12}\right): \hat{Y}_{11 j}^{2}+\hat{Y}_{12 j}^{2}=\tau_{j}^{2}\left(\psi_{j}^{(0)}\left(t_{0}, v\right)\right)^{2}, \hat{Y}_{11 k}^{2}\right. \\
& \left.+\widehat{Y}_{12 k}^{2}<\tau_{k}^{2}\left(\psi_{k}^{(0)}\left(t_{0}, \nu\right)\right)^{2}, k=\overline{1, p}, k \neq j, \nu \in\left(\nu_{1}, \nu_{2}\right)\right\} .
\end{aligned}
$$


The behaviour of the integral curves of system (13) is studied on the surface $\partial \widehat{\Omega}_{j}\left(\tau, \varphi^{(0)}\left(z\left(t_{0}, \nu\right)\right)\right)$, for fixed $j \in\{1, \ldots, p\}$.

According to the condition, the matrix $P(z)$ has the property $S_{3}$, and the vector function $F\left(z, Y(z), Y^{\prime}(z)\right)$ has the property $M_{3}$ with respect to the vector function $\varphi^{(0)}(z)$. Therefore,

$$
\left(t_{0}^{r_{0}-1} \bar{T}, \frac{\bar{N}_{j}}{2}\right) \sim \sqrt{\left(\hat{p}_{j j 1}(\nu)\right)^{2}+\left(\hat{p}_{j j 2}(\nu)\right)^{2}} \cdot \sin \left((r-1) \nu+\hat{\alpha}_{j j}(\nu)\right), j=\overline{1, p},
$$

for $t_{0} \rightarrow+0, \nu \in\left(v_{1}, \nu_{2}\right)$. Hence,

$$
\operatorname{sign}\left(t_{0}^{r_{0}-1} \bar{T}, \frac{\bar{N}_{j}}{2}\right)=\operatorname{sign}\left(\sin \left((r-1) \nu+\hat{\alpha}_{j j}(v)\right)\right), j=\overline{1, p}, \nu \in\left(\nu_{1}, \nu_{2}\right) .
$$

Without restricting the generality, for every fixed $t_{0} \in\left(0, t^{*}\right)$ the surface $\partial \widehat{\Omega}\left(\tau, \varphi^{(0)}\left(z\left(t_{0}, v\right)\right)\right) \in \Lambda_{+. k}\left(t^{*}\right), k \in\{+,-\}$ is a surface without contact for system (13).

Since system (9) belongs to the class $C_{+. k}, k \in\{+,-\}$, any integral curve of system (13), passing through a point of the set $\widehat{\Omega}\left(\tau, \varphi^{(0)}\left(z\left(t_{0}, \nu\right)\right)\right) \cap\left(\nu=v_{0}\right), \nu_{0} \in\left(v_{1}, \nu_{2}\right)$, for $\left(t_{0}, v_{0}\right) \in \Lambda_{+.+}\left(t^{*}\right)$, stays in the domain $\widehat{\Omega}\left(\tau, \varphi^{(0)}\left(z\left(t_{0}, \nu\right)\right)\right)$ as $\nu$ decreases, and for $\left(t_{0}, \nu_{0}\right) \in \Lambda_{+.-}\left(t^{*}\right)$, stays in the domain $\widehat{\Omega}\left(\tau, \varphi^{(0)}\left(z\left(t_{0}, \nu\right)\right)\right)$ as $\nu$ increases.

Moreover, the following inequalities are true

$$
\left|Y_{1 s j}\left(z\left(t_{0}, \nu\right)\right)\right|^{2}<\tau_{j}^{2}\left|\psi_{j}^{(0)}\left(t_{0}, \nu\right)\right|^{2}, j=\overline{1, p}, s=1,2 \text {, }
$$

$\left(t_{0}, \nu\right) \in \Lambda_{+. k}\left(t^{*}\right), k \in\{+,-\}$. 
(3) Now we apply the method of analytic extension for problems resolved with respect to the derivatives proposed by Grabovskaya [1]. This method was developed for problems unsolved with respect to the derivatives by Samkova [6, 7] and used by Limanska and Samkova [3] to prove item 3 of Theorem 2 in [3]. Let's assume that the vectors $\delta, \tau \in \mathbb{C}^{p}, \delta_{j} \neq 0, \tau_{j} \neq 0, j=\overline{1, p}$ satisfy the inequality

$$
\delta_{j}^{2}<\tau_{j}^{2}, j=\overline{1, p}
$$

In item (1) of the proof of Theorem 1, it was established that, along the curve $L_{\nu_{0}}\left(t_{1}\right), \nu_{0} \in\left(\nu_{1}, \nu_{2}\right)$ for $t \in\left(0, t^{*}\right)$ there exist the infinite numbers of continuously differentiable solutions of system (9), there exist infinite numbers of continuously differentiable solutions of system (15). The set of these solutions is denoted by $\left\{Y_{1}\left(z\left(t, \nu_{0}\right)\right)\right\}$.

Any solution $Y_{1}\left(z\left(t, v_{0}\right)\right)$ from the set $\left\{Y_{1}\left(z\left(t, \nu_{0}\right)\right)\right\}$ can be analytically extended from $L_{\nu_{0}}\left(t_{1}\right)$, where $(t, v) \in \Lambda_{+. k}\left(t^{*}\right)$, for fixed $\nu_{0} \in\left(\nu_{1}, \nu_{2}\right) o$ a domain containing it with preservation of estimate (17).

It follows from item (2) of the theorem that, in the case where inequality (18) is true, the solution $Y_{1}\left(z\left(t, v_{0}\right)\right)$, for fixed $\nu=\nu_{0}$, can be extended from the curve $L_{\nu_{0}}\left(t_{1}\right)$ along curves $O_{t_{1}}\left(t_{0}\right)$ to the set $\widehat{\Omega}\left(\tau, \varphi^{(0)}\left(z\left(t^{*}, v\right)\right)\right)$ on the condition that $t \in\left(0,\left|z\left(t_{0}, v\right)\right|\right]$. Moreover, the analytic extension is denoted by $Y_{1}(z)$. Hence, we obtain the set of solutions of the system (9) as $\left\{Y_{1}(z)\right\}$.

Thus, any solution of the system (9) - $Y_{1}(z)$ can be analytically extended to $G_{+. k}\left(t^{*}\right) \times\left\{Y:\left|Y_{1 j}\right|<\delta_{j}\left|\varphi_{j}\left(z\left(t_{0}, \nu\right)\right)\right|, j=\overline{1, p}\right\}$. Moreover, inequalities (14) are true in this domain. Theorem 1 is proved. 
Let us suppose that the system (1) can be reduced to the form (3). For $Y_{2} \in H_{r}^{n-p}$, the system (3) can be reduced to the system (9). We investigate the problems of existence and asymptotic behaviour of the solutions of the system (3) that satisfy the initial condition (6), and additional condition (7) for $Y_{2} \in H_{r}^{n-p}$.

Theorem 2. Let us suppose that $p<n, A(z)$ is analytic matrix in the domain $D_{1}$ and $\operatorname{rang} A(z)=p$ on the conditions that $z \in D_{1}$. Moreover, suppose that the system (1) can be reduced to the form (3), and the system (3) on the condition that $Y_{2} \in H_{r}^{n-p}$ can be reduced to the form (9). The conditions (1)-(3) of Theorem 1 are satisfied for system (9).

Then, for each $k \in\{+,-\}$, for some $t^{*} \in\left(0, t_{1}\right)$ and for each $Y_{2} \in H_{r}^{n-p}$ there exist such solutions of the system $\left({ }^{*} I\right) Y(z)=\left(Y_{11}(z)\right.$, $\left.\ldots, Y_{1 p}(z), Y_{21}(z), \ldots, Y_{2 n-p}(z)\right)$, for which the first $p$ components are analytic in the domain $D_{1} \cap G_{+. k}\left(t^{*}\right)$ and admit estimate (14) in this domain.

Proof. According to the Theorem 1, the solution $Y_{1}(z)$ of the system (9) is analytically extended to $G_{+. k}\left(t^{*}\right) \times\left\{Y:\left|Y_{1 j}\right|<\delta_{j}\left|\varphi_{j}(z)\right|, j=\overline{1, p}\right\}$, and besides, the solution $Y_{1}(z)$ admits estimate (14) in this domain. Thus, the system (1), for some fixed function $Y_{2} \in H_{r}^{n-p}$, has at least one solution $Y=\left(Y_{1}(z), Y_{2}(z)\right)$, for which the first $p$ components are analytic in the domain $G_{+. k}\left(t^{*}\right) \times\left\{Y:\left|Y_{1 j}\right|<\delta_{j}\left|\varphi_{j}(z)\right|, j=\overline{1, p}\right\}$ and admit estimate (14) on the condition that $z \in D_{1} \cap G_{+. k}\left(t^{*}\right)$. Theorem 2 is proved. 


\section{Conclusion}

The necessary conditions of the existence of the analytical solutions for the system of differential equations (9), partially solved relatively to the derivatives, with square matrices, in the presence of a pole $z=0$, were found. It was found an estimate for these solutions in the neighbourhood of the point $z=0$.

The theorem of the existence of the solutions for the system (1) was proved. The first $p$ components of these solutions are analytic in the neighbourhood of the point $z=0$, the rest $n-p$ components are from the class of analytic in the domain $D_{10}$ functions that have the pole in the point $z=0$.

\section{References}

[1] R. G. Grabovskaya, The asymptotic behavior of the solution of a system of two first order nonlinear differential equations, Differentsial'nye Uravneniya 11(4) (1975), 639-644.

[2] R. G. Grabovskaya and J. Diblic, Asymptotic of Systems of Differential Equations Unsolved with Respect to the Derivatives [in Russian], Dep. at VINITI. 1986.

[3] D. Limanska and G. Samkova, About behavior of solutions of some systems of differential equations, which is partially resolved relatively to the derivatives, Bulletin of Mechnikov's Odessa National University 19(1) (2014), 16-28.

[4] D. Limanska, About behavior of solutions of some systems of differential equations, which is partially resolved relatively to the derivatives in the case of a pole, Nonlinear Oscillations 20(1) (2017), 113-126.

[5] D. E. Limanska and G. E. Samkova, On the existence of analytic solutions of certain types of system, partially resolved relatively to the derivatives, in the case of a pole, Memoirs on Differential Equations and Mathematical Physics 74 (2018), 113-124.

[6] G. E. Samkova, Existence and asymptotic behavior of the analytic solutions of some singular differential systems, unsolved with respect to the derivatives, Differentsial'nye Uravneniya 27(11) (1991), 2012-2013.

[7] G. E. Samkova and N. V. Sharay, On the investigation of one semi explicit system of differential equations in the case of a variable pencil of matrices, Nonlinear Oscillations 5(2) (2002), 224-236. 
[8] A. M. Samoilenko, On the asymptotic integration of a system of linear differential equations with a small parameter in the coefficients of a part of derivatives, Ukr. Mat. Zh 54(11) (2002), 1505-1516.

[9] N. V. Sharai and H. E. Samkova, Asymptotic of solutions of some semiexplicit systems of differential equations, Nauk. Visn. Cherniv. Univ. Ser. Mat., Issue 314-315 (2006), 1810-188.

[10] I. Shkil', I. Starun and V. Yakovets, Asymptotic Integration of Linear Systems of Ordinary Differential Equations with Degenerations [in Russian], Vyshcha Shkola Kiev, 1991.

[11] J. Diblic, One theorem on asymptotic behavior of solutions of a certain system of quasilinear differential equations not solved with respect to derivatives, Riv. Mat. Univ. Parma, 13 (1987), 413-419.

[12] M. Jwano, A method to construct stable domain of a sectorial type, Funkc. Ekvacioj 42(1) (1999), 71-103.

[13] O. Song Guk, Pak Ponk and Chol Permissible, Boundary condition of a system of linear ordinary differential equations in a closed angle domain of complex plane, Kwahakwon Thongbo Bull. Acad. Sci. DPR Korea 3 (2001), 2-4. 